\title{
SOLVING OVERDETERMINED EIGENVALUE PROBLEMS
}

\author{
SAPTARSHI DAS* AND ARNOLD NEUMAIER ${ }^{\dagger}$
}

\begin{abstract}
We propose a new interpretation of the generalized overdetermined eigenvalue problem $(\mathbf{A}-\lambda \mathbf{B}) \mathbf{v} \approx 0$ for two $m \times n(m>n)$ matrices $\mathbf{A}$ and $\mathbf{B}$, its stability analysis, and an efficient algorithm for solving it. Usually, the matrix pencil $\{\mathbf{A}-\lambda \mathbf{B}\}$ does not have any rank deficient member. Therefore we aim to compute $\lambda$ for which $\mathbf{A}-\lambda \mathbf{B}$ is as close as possible to rank deficient; i.e., we search for $\lambda$ that locally minimize the smallest singular value over the matrix pencil $\{\mathbf{A}-\lambda \mathbf{B}\}$. Practically, the proposed algorithm requires $\mathcal{O}\left(m n^{2}\right)$ operations for computing all the eigenpairs. We also describe a method to compute practical starting eigenpairs. The effectiveness of the new approach is demonstrated with numerical experiments. A MATLAB based implementation of the proposed algorithm can be found at:

http://www.mat.univie.ac.at/ neum/software/oeig/
\end{abstract}

Key words. Overdetermined eigenvalue problem, numerical linear algebra.

AMS subject classifications. 45C05, 65F $30,65 \mathrm{~F} 22$

\section{Introduction.}

1.1. Motivation. There are several applications where the overdetermined eigenvalue problem $(\mathbf{A}-\lambda \mathbf{B}) \mathbf{v} \approx 0, \mathbf{v} \neq 0$ arise naturally; typically when the matrices $\mathbf{A}$ and $\mathbf{B}$ are constructed row wise where more rows can be obtained by additional measurements or simulations. For practical applications that benefit from the solution of an overdetermined eigenvalue problem, see SARKAR and PEREIRA 2002 [22, Ouibrahim 2002 [20, Neugebauer 2008 [19, Alharbi 2010 [1]. Specific applications include the blind detection of signals, see LAU et al. 2002 [16], and the harmonic inversion problem, see Roy and KaIlath 1989 21], HUA and SARKar 2002 [15.

In such practical problems, the entries of the matrices $\mathbf{A}$ and $\mathbf{B}$ comes from noisy measurements of a certain process. Generally for such applications, the physical process that generated the data suggests in theory the existence of a solution or a set of solutions. However, because of the noise in the measured matrices $\mathbf{A}$ and $\mathbf{B}$, the matrix pencil $\{\mathbf{A}-\lambda \mathbf{B}\}$ might not admit any rank deficient member. By solving the proposed overdetermined eigenvalue problem, and thereby utilizing the additional information, one expects to reach a more stable result, which accurately reconstructs the parameters of the process.

Below we provide two applications that can benefit from the proposed formulation and algorithm of the overdetermined eigenvalue problem.

Harmonic inversion: Several problems related to communication systems, object tracking and many other applications require harmonic inversion. In the framework of the harmonic inversion problem [14, the model for the data collected at

${ }^{*}$ Faculty of Mathematics, University of Vienna (saptarshi.das@univie.ac.at). Supported by the Federal Ministry of Science and Research, Austria, in the framework of the Austrian in-kind contribution to the European Southern Observatory (ESO).

${ }^{\dagger}$ Faculty of Mathematics, University of Vienna. (arnold.neumaier@univie.ac.at) 
equi-spaced discrete time point is given by:

$$
y_{k}=x_{k}+\eta_{k}=\sum_{m=1}^{M} b_{m} z_{m}^{k}+\eta_{k}, \quad k=0, \ldots K-1,
$$

here $x_{k}$ and $\eta_{k}$ denote the noise-free and noise components of the observation $y_{k}$, respectively, $M$ denotes the order of the model, and $K$ is the total number of samples. The parameters $b_{m}$ are linear, and easy to estimate once an estimate of the nonlinear parameters $z_{m}$ are available. In the noiseless case $\left(y_{k}=x_{k}\right)$, the $z_{m}$ can be computed as an eigenvalue of the following overdetermined eigenvalues problem:

$$
\mathbf{A v}=\lambda \mathbf{B v} .
$$

where $\mathbf{A}$ and $\mathbf{B}$ are two Hankel matrices constructed from the $x_{k}$. For details regarding how such Hankel matrices are constructed, see 14. However in the presence of noise, i.e., when the Hankel matrices $\mathbf{A}$ and $\mathbf{B}$ are constructed from the measured $y_{k}$, the matrix pencil $\{\mathbf{A}-\lambda \mathbf{B}\}$ might not admit any rank deficient member. There is a large literature on this problem, where the problem is treated in different ad hoc approaches. We believe that the noisy case benefits from the proposed formulation and algorithm for the overdetermined eigenvalue problem. Further details will be provided elsewhere.

Distance to uncontrollability: An accurate method for solving overdetermined eigenvalue problems is also beneficial for estimating the distance to uncontrollability of a linear control system. It is shown by EIsing [6] that for a linear control system

$$
\dot{\mathbf{x}}=\mathbf{A}_{1} \mathbf{x}+\mathbf{A}_{2} \mathbf{u}
$$

the distance to uncontrollability is given by

$$
\rho=\min _{\lambda} \sigma_{\min }\left(\left[\mathbf{A}_{1}-\lambda \mathbf{I}, \mathbf{A}_{2}\right]\right)
$$

here, $\mathbf{x} \in \mathbb{C}^{n_{1} \times 1}$ is the state variable, $\mathbf{u} \in \mathbb{C}^{n_{2} \times 1}$ is the control variable, $\mathbf{A}_{\mathbf{1}} \in \mathbb{C}^{n_{1} \times n_{1}}$, $\mathbf{A}_{2} \in \mathbb{C}^{n_{1} \times n_{2}}$ are the system matrices, and $\sigma_{\min }(\mathcal{G})$ denotes the smallest singular value of the matrix $\mathcal{G}$. Equivalently, the distance to uncontrollability is measured by solving an overdetermined eigenvalue problem with the following pair of matrices:

$$
\mathbf{A}=\left[\begin{array}{c}
\mathbf{A}_{1}^{*} \\
\mathbf{A}_{2}^{*}
\end{array}\right] \text { and } \mathbf{B}=\left[\begin{array}{c}
\mathbf{I}_{n_{1} \times n_{1}} \\
\mathbf{0}_{n_{2} \times n_{2}}
\end{array}\right]
$$

However, it should be noted that distance to uncontrollability is given by global minimum of equation (1.4). On the other hand, in the framework of overdetermined eigenvalue problems, the objective is to locate all the local minima. In fact, the proposed algorithm computes much more spectral information, which is discarded when only the distance to uncontrollability is required.

1.2. Previous Work. The generalized eigenvalue problem for square matrices is well studied over last five decades. In 1973, Moler and Stewart 18, proposed the well known $Q Z$ decomposition, also referred to as the generalized Schur decomposition, for computing generalized eigenpairs. In 1971, DeLL et al. 44 proposed a method to compute generalized eigenpairs of rectangular matrices. This algorithm is applicable to matrix pairs $(\mathbf{A}, \mathbf{B})$ which admits a non-trivial solution for 
$(\mathbf{A}-\lambda \mathbf{B}) \mathbf{v}=0, \mathbf{v} \neq 0$, and therefore applicable only in very special noiseless cases. On the other hand, the generalized eigenvalue problem for overdetermined matrices which might not admit any non-trivial solution, is a matter of current interest.

Most of the work related to the generalized eigenvalue problem for overdetermined matrices concentrates on theoretical analysis. In [2, 23, 5, 7] the distance to the closest matrix pair which admit a non-trivial eigenpairs is studied. WRIGHT and TREFETHEN [26] studied the pseudospectra of overdetermined matrix pencils.

To our knowledge, the only algorithm available so far for the overdetermined eigenvalue problem with noisy measurements is by Boutry et al. 2006, 3. Their method is based on minimal perturbation approach (MPA), where the objective is to estimate the closest matrix pair $\left(\mathbf{A}_{0}, \mathbf{B}_{0}\right)$ to the given matrix pair $(\mathbf{A}, \mathbf{B})$ in Frobenius norm, such that $\left(\mathbf{A}_{0}, \mathbf{B}_{0}\right)$ admits a non-trivial eigenpair. Finally, the eigenpairs corresponding to $\left(\mathbf{A}_{0}, \mathbf{B}_{0}\right)$ are considered as the estimated eigenpairs for the given matrix pair $(\mathbf{A}, \mathbf{B})$. Throughout the remainder of this paper, we refer to this approach as the MPA. Further extensions of the MPA for matrices with a special structure are studied in [17.

In [3] it is proved that the MPA approach is equivalent to solving the following optimization problem:

$$
\{\hat{\lambda}, \hat{\mathbf{v}}\}=\underset{\{\lambda, \mathbf{v}\}}{\operatorname{argmin}} g(\lambda, \mathbf{v})=\underset{\{\lambda, \mathbf{v}\}}{\operatorname{argmin}} \frac{\|(\mathbf{A}-\lambda \mathbf{B}) \mathbf{v}\|^{2}}{1+|\lambda|^{2}} ; \text { subject to: }\|\mathbf{v}\|=1 .
$$

We note that, ignoring the eigenvector $\mathbf{v}$, the optimization problem (1.6) is equivalent to:

$$
\hat{\lambda}=\underset{\lambda}{\operatorname{argmin}} g_{0}(\lambda)=\underset{\lambda}{\operatorname{argmin}} \frac{\sigma_{\min }(\mathbf{A}-\lambda \mathbf{B})}{\sqrt{1+|\lambda|^{2}}},
$$

where the function $\sigma_{\min }$ returns the smallest singular value of its matrix argument.

In the context of linear control problems, the first polynomial time algorithm for computing the distance to uncontrollability was proposed by GU 2000 [10]. Later, GU published a faster method in 2007 [11. These methods by Gu are based on bisection. Other methods for computing the distance to uncontrollability includes [8], [12]. For a detailed discussion of the sensitivity of computational control problems see HiGHAM et al. 2004 [13.

1.3. Contributions. In this paper, we propose a new interpretation for overdetermined generalized eigenvalue problems, an associated stability analysis, and an efficient algorithm for solving the problem, given two matrices $\mathbf{A}$ and $\mathbf{B}$ of size $m \times n$ $(m>n)$. The overdetermined eigenvalue problem might not admit any eigenpair $\lambda, \mathbf{v}$ for which $(\mathbf{A}-\lambda \mathbf{B}) \mathbf{v}=0, \mathbf{v} \neq 0$, i.e., the matrix pencil $\{\mathbf{A}-\lambda \mathbf{B}\}$ might not admit any rank deficient member. Therefore, we reformulate the generalized eigenvalue problem as the following optimization problem:

$$
\{\hat{\lambda}, \hat{\mathbf{v}}\}=\underset{\lambda, \mathbf{v} \neq 0}{\operatorname{argmin}} f(\lambda, \mathbf{v})=\underset{\lambda, \mathbf{v} \neq 0}{\operatorname{argmin}} \frac{\|(\mathbf{A}-\lambda \mathbf{B}) \mathbf{v}\|^{2}}{\|\mathbf{D v}\|^{2}},
$$

where, $\lambda \in \mathbb{C}, \mathbf{v} \in \mathbb{C}^{n},\|\cdot\|$ denotes the Euclidean norm of its vector argument, and $\mathbf{D}$ is a non singular diagonal scaling matrix, typically with entries proportional to the entries of the corresponding rows of $\mathbf{A}-\lambda \mathbf{B}$. The scaling using matrix $D$ is a pre-processing step, in case all the components of $\mathbf{v}$ have the same natural scale the 
scaling is not required. After reinstating $\mathbf{A} \leftarrow \mathbf{A} \mathbf{D}^{-1}, \mathbf{B} \leftarrow \mathbf{B D}^{-1}$ and $\mathbf{v} \leftarrow \mathbf{D v}$, we get:

$$
\{\hat{\lambda}, \hat{\mathbf{v}}\}=\underset{\lambda, \mathbf{v} \neq 0}{\operatorname{argmin}} f(\lambda, \mathbf{v})=\underset{\lambda, \mathbf{v} \neq 0}{\operatorname{argmin}} \frac{\|(\mathbf{A}-\lambda \mathbf{B}) \mathbf{v}\|^{2}}{\|\mathbf{v}\|^{2}},
$$

We note that ignoring the eigenvector $\mathbf{v}$, the optimization problem (1.9) is equivalent to:

$$
\hat{\lambda}=\underset{\lambda}{\operatorname{argmin}} f_{0}(\lambda)=\underset{\lambda}{\operatorname{argmin}} \sigma_{\min }(\mathbf{A}-\lambda \mathbf{B}),
$$

where the function $\sigma_{\min }$ returns the smallest singular value of its matrix argument. Our aim is to find all the local minima, and we refer to each local minimum as an overdetermined eigenvalue.

Along with the new interpretation of overdetermined eigenvalue, our contribution in this paper includes:

- conditions under which the overdetermined eigenvalues are stable.

- an efficient algorithm to compute all the overdetermined eigenpairs with a computational complexity of $\mathcal{O}\left(m n^{2}\right)$.

- practical starting points for the algorithm.

- several experiments that validates the proposed interpretation and the algorithm for computing overdetermined eigenpairs.

As described in equation (1.4), the proposed method for solving overdetermined eigenvalue problems also allows one to compute the distance to uncontrollability for a linear control system. In terms of computational complexity, the fastest method available so far is proposed by Gu et al. [1], and this method requires $\mathcal{O}\left(n^{4}\right)$ operations. On the other hand, our empirical evidence suggest that the proposed method can compute all the local minima exhaustively in $\mathcal{O}\left(n^{3}\right)$ operations. Consequently, the empirical complexity to compute the distance to uncontrollability using the proposed method is $\mathcal{O}\left(n^{3}\right)$ operations.

Solving the optimization problem posed in equations (1.9) and (1.10) instead of the MPA optimization problem posed in equations (1.6) and (1.7) has several advantages. The advantages are explained, and demonstrated numerically in Section 4.

1.4. Organization. This paper is organized as follows: in Section 2 we deduce the conditions under which the overdetermined eigenpairs are stable. In Section 3, we introduce algorithms to estimate the generalized eigenpairs for overdetermined matrices. In that section, the algorithms, computational complexity, and implementation details of the proposed methods are also described. Results from our numerical experiments are presented in Section 4 In that section, we also demonstrate procedures to simulate overdetermined matrix pairs with prescribed eigenpairs. A brief discussion on the relation between overdetermined eigenvalues and pseudospectra is presented in Section 5. Our conclusions are presented in Section 6 .

The web-page: http://www.mat.univie.ac.at/ neum/software/oeig/

contains a well documented software consisting of all the algorithms proposed in this paper, as well as algorithms proposed by other researchers, utility functions to simulate overdetermined matrices with specified eigenpairs, and tools for visualization.

2. Stability of Overdetermined Eigenvalues. In this section, we discuss the stability of the overdetermined eigenpairs as introduced in (1.9). We do this 
by showing that, under mild conditions, the generalized eigenvalues and generalized eigenvectors depend continuously differentiably on the data, with computable partial derivatives that specify the sensitivity with respect to the data. Such stability analysis is necessary to guarantee that under mild conditions the eigenpairs as formulated in equation (1.8) are well-determined even if there are small error in the data i.e., matrices $\mathbf{A}$ and $\mathbf{B}$. In practice, the matrices $\mathbf{A}$ and $\mathbf{B}$ always have some amount of error. The error in the data matrices $\mathbf{A}$ and $\mathbf{B}$ can be measurement error, round-off error etc...

For the sake of notational convenience we introduce the matrix valued polynomial

$$
\mathbf{P}(\lambda)=\mathbf{A}-\lambda \mathbf{B}, \quad \lambda \in \mathbb{C} .
$$

Consequently, the derivative of $\mathbf{P}$ with respect to $\lambda$ is: $\mathbf{P}^{\prime}(\lambda)=-\mathbf{B}$. Using the above notation, the objective function of the unconstrained optimization problem posed to solve the overdetermined eigenvalue problem (1.9) may be expressed as

$$
f(\lambda, \mathbf{v})=\frac{\|\mathbf{P}(\lambda) \mathbf{v}\|^{2}}{\|\mathbf{v}\|^{2}} .
$$

$\mathbf{Y}(\lambda)$, and $\mathbf{Z}(\lambda)$, We shall also need the matrix valued polynomials defined by

$$
\begin{aligned}
& \mathbf{X}(\lambda)=\mathbf{P}(\lambda)^{*} \mathbf{P}(\lambda) \\
& \mathbf{Y}(\lambda)=\mathbf{P}(\lambda)^{*} \mathbf{P}^{\prime}(\lambda) \\
& \mathbf{Z}(\lambda)=\mathbf{X}-\mathbf{I} f+\gamma \mathbf{v} \mathbf{v}^{*}
\end{aligned}
$$

where $\gamma$ is a real scalar.

THEOREM 2.1. An overdetermined eigenpair $(\lambda, \mathbf{v})$ (with $\|v\|=1$ ) of two matrices $\mathbf{A}$ and $\mathbf{B}$ is stable if the following two conditions (C1) and (C2) hold for some real scalar $\gamma$ :

(C1) $\left|\mathbf{v}^{*} \mathbf{Y} \mathbf{Z}^{-1} \mathbf{X v}\right|^{2}-\left|\mathbf{v}^{*} \mathbf{X} \mathbf{Z}^{-1} \mathbf{Y} \mathbf{v}\right|^{2}$ is bounded away from zero.

(C2) If $\sigma_{i}$ are the singular values of $\mathbf{X}-\mathbf{I} f$ then $\sigma_{n-1}>\sigma_{n}$ and the number

$$
q:=\frac{\sigma_{1}-\sigma_{n}}{\sigma_{n-1}-\sigma_{n}}
$$

is reasonably bounded.

Proof. At a minimizer $(\lambda, \mathbf{v})$ of (2.2), $f$ is stationary with respect to $\lambda$ and $\mathbf{v}$. By setting $\frac{\partial f}{\partial \lambda}$ and $\frac{\partial f}{\partial \mathbf{v}}$ to zero, we get the equations

$$
\begin{gathered}
\mathbf{v}^{*} \mathbf{P}(\lambda)^{*} \mathbf{P}^{\prime}(\lambda) \mathbf{v}=0, \\
\mathbf{P}(\lambda)^{*} \mathbf{P}(\lambda) \mathbf{v}=f(\lambda, \mathbf{v}) \mathbf{v} .
\end{gathered}
$$

Since $f$ is homogeneous in $\mathbf{v}$, we may require $\mathbf{v}$ to be normalized, i.e.,

$$
\mathbf{v}^{*} \mathbf{v}=1 .
$$

Using (2.3), (2.4), we may write the above conditions as

$$
\begin{aligned}
\mathbf{v}^{*} \mathbf{Y}(\lambda) \mathbf{v} & =0, \\
\mathbf{X}(\lambda) \mathbf{v}-f \mathbf{v} & =0, \\
\mathbf{v}^{*} \mathbf{v}-1 & =0 .
\end{aligned}
$$


Now we suppose that the data, i.e., the matrices $\mathbf{A}$ and $\mathbf{B}$, depend smoothly on a parameter $\tau$. The eigenpair is stable if the solution $f(\tau), \lambda(\tau), \mathbf{v}(\tau)$ is smooth in $\tau$. In other words, we need $\dot{f}=\frac{d f}{d \tau}, \dot{\lambda}=\frac{d \lambda}{d \tau}$ and $\dot{\mathbf{v}}=\frac{d \mathbf{v}}{d \tau}$ to be well-defined and reasonably bounded. We now derive explicit formulas for these derivatives.

Differentiating $\mathbf{X}$ and $\mathbf{Y}$ with respect to $\tau$ gives

$$
\begin{aligned}
\frac{d}{d \tau} \mathbf{X}(\lambda) & =\mathbf{X}_{\tau}(\lambda)+\mathbf{X}(\lambda) \frac{d \lambda}{d \tau}, \\
\frac{d}{d \tau} \mathbf{Y}(\lambda) & =\mathbf{Y}_{\tau}(\lambda)+\mathbf{Y}(\lambda) \frac{d \lambda}{d \tau},
\end{aligned}
$$

where $\mathbf{X}_{\tau}$ and $\mathbf{Y}_{\tau}$ are respectively the partial derivative of $\mathbf{X}$ and $\mathbf{Y}$ with respect to $\tau$. We shall show how to satisfy the equations

$$
\begin{array}{r}
\dot{\mathbf{v}}^{*} \mathbf{Y} \mathbf{v}+\mathbf{v}^{*}\left(\mathbf{Y}_{\tau}+\dot{\lambda} \mathbf{Y}\right) \mathbf{v}+\mathbf{v}^{*} \mathbf{Y} \dot{\mathbf{v}}=0 \\
\left(\mathbf{X}_{\tau}+\dot{\lambda} \mathbf{X}\right) \mathbf{v}+\mathbf{X} \dot{\mathbf{v}}-\dot{f} \mathbf{v}-\dot{\mathbf{v}} f=0 \\
\mathbf{v}^{*} \dot{\mathbf{v}}=0
\end{array}
$$

It is easy to see that these imply the equation

$$
\frac{d}{d \tau}\left(\begin{array}{c}
\mathbf{v}^{*} \mathbf{Y}(\lambda) \mathbf{v} \\
\mathbf{X}(\lambda)-f \mathbf{v} \\
\mathbf{v}^{*} \mathbf{v}
\end{array}\right)=\mathbf{0} .
$$

Consequently, a solution of the system of equations (2.15), (2.16), (2.17) near $\tau=0$ gives a curve of overdetermined eigenpairs that is smooth in $\tau$, proving our claim.

Using the definition of $\mathbf{Z}(2.5)$ and the relations (2.16) and (2.17), we get

$$
\dot{\mathbf{v}}=\mathbf{Z}^{-1}\left(\dot{f} \mathbf{v}-\mathbf{X}_{\tau} \mathbf{v}-\dot{\lambda} \mathbf{X} \mathbf{v}\right) .
$$

Since $\mathbf{Z}$ is a Hermitian matrix and $\mathbf{Z} \mathbf{v}=\gamma \mathbf{v}$, we can write

$$
\gamma \mathbf{v}^{*} \mathbf{Z}^{-1}=\mathbf{v}^{*}
$$

Since (2.11) implies

$$
\mathbf{v}^{*} \mathbf{X} \mathbf{v}=f \mathbf{v}^{*} \mathbf{v}=f
$$

we get, using the relations (2.17), (2.19), (2.20), and (2.21),

$$
\begin{aligned}
\dot{f}-\mathbf{v}^{*} \mathbf{X}_{\tau} \mathbf{v}-\dot{\lambda} f & =\mathbf{v}^{*}\left(\dot{f} \mathbf{v}-\mathbf{X}_{\tau} \mathbf{v}-\dot{\lambda} \mathbf{X} \mathbf{v}\right) \\
& =\gamma \mathbf{v}^{*} \mathbf{Z}^{-1}\left(\dot{f} \mathbf{v}-\mathbf{X}_{\tau} \mathbf{v}-\dot{\lambda} \mathbf{X} \mathbf{v}\right) \\
& =\gamma \mathbf{v}^{*} \dot{\mathbf{v}}=0
\end{aligned}
$$

Thus we can express $\dot{f}$ as

$$
\dot{f}=\dot{\lambda} f+\mathbf{v}^{*} \mathbf{X}_{\tau} \mathbf{v} .
$$

Starting from condition (2.15), and using the relations (2.19), and (2.10) we get

$$
\left(\dot{f} \mathbf{v}-\mathbf{X}_{\tau} \mathbf{v}-\dot{\lambda} \mathbf{X} \mathbf{v}\right)^{*} \mathbf{Z}^{-1} \mathbf{Y} \mathbf{v}+\mathbf{v}^{*} \mathbf{Y}_{\tau} \mathbf{v}+\mathbf{v}^{*} \mathbf{Y} \mathbf{Z}^{-1}\left(\dot{f} \mathbf{v}-\mathbf{X}_{\tau} \mathbf{v}-\dot{\lambda} \mathbf{X} \mathbf{v}\right)=0 .
$$

We may eliminate $\dot{f}$ from the above equation using relation (2.20), and get

$$
\left(\mathbf{X}_{\tau} \mathbf{v}+\dot{\lambda} \mathbf{X} \mathbf{v}\right)^{*} \mathbf{Z}^{-1} \mathbf{Y} \mathbf{v}-\mathbf{v}^{*} \mathbf{Y}_{\tau} \mathbf{v}+\mathbf{v}^{*} \mathbf{Y} \mathbf{Z}^{-1}\left(\mathbf{X}_{\tau} \mathbf{v}+\dot{\lambda} \mathbf{X} \mathbf{v}\right)=0 .
$$


From this equation, $\dot{\lambda}$ can be computed explicitly: If we denote the coefficients of $\dot{\lambda}^{*}$ by $a$, the coefficients of $\dot{\lambda}$ by $b$, and the terms independent of $\dot{\lambda}$ (as well as independent of $\dot{f}, \dot{\mathbf{v}}$ ) by $c$, then $\dot{\lambda}$ is found to be

$$
\dot{\lambda}=\frac{b^{*} c-c^{*} a}{b^{*} b-a^{*} a} .
$$

By construction of $a$ and $b$ we have $b^{*} b-a^{*} a=\left|\mathbf{v}^{*} \mathbf{Y} \mathbf{Z}^{-1} \mathbf{X} \mathbf{v}\right|^{2}-\left|\mathbf{v}^{*} \mathbf{X} \mathbf{Z}^{-1} \mathbf{Y v}\right|^{2}$. Thus the condition $(\mathbf{C} 1)$ guarantees a well-defined and reasonably bounded $\dot{\lambda}$. Equation (2.23) now shows that $\dot{f}$ is also well-defined and reasonably bounded.

Finally, equation (2.19) shows that $\dot{\mathbf{v}}$ is well-defined and reasonably bounded if, in addition, $\mathbf{Z}$ is well conditioned. This is guaranteed by condition (C2) if we choose $\gamma$ appropriately. Indeed, if $\gamma \in\left[\sigma_{n-1}-\sigma_{n}, \sigma_{1}-\sigma_{n}\right]$, then

$$
\operatorname{cond}(\mathbf{Z})=\frac{\sigma_{1}-\sigma_{n}}{\sigma_{n-1}-\sigma_{n}}=q
$$

(It is easy to see that this choice of $\gamma$ minimizes the condition number of $\mathbf{Z}$.)

REMARK 1. An overdetermined eigenvalue problem may also have illposed eigenspaces. For such a case, the conditions $(\mathbf{C 1})$ and $(\mathbf{C 2})$ are not satisfied. In principle, these two conditions are computable, though their cost is an order of magnitude larger than the computation of the spectral information itself. The complexity of computing the spectral information is described in Section 3 .

\section{Algorithms for overdetermined eigenvalue problem.}

3.1. Direct Approach. We consider the problem to find all the local minima of the function $f$ defined in equation (1.9). One may impose an equivalent constraint $\|\mathbf{v}\|=1$ to remove the scale invariance. The function $f$ is multi-modal and has at most $n$ local minima, unless the matrix pencil $\{\mathbf{A}-\lambda \mathbf{B}\}$ is identically rank deficient.

We first show that it is possible to find a unitary transformation that reduces the objective function $f$ in equation (1.9) to an equivalent form that consists of upper triangular matrices and a symmetric positive definite matrix.

THEOREM 3.1. For a given pair of matrices $(\mathbf{A}, \mathbf{B})$ of size $m \times n(m \geqslant n)$, there exists upper triangular matrices $\mathbf{R}_{0}, \mathbf{R} \in \mathbb{C}^{n \times n}$, such that

$$
\underset{\lambda, \mathbf{v} \neq 0}{\operatorname{argmin}} \frac{\|(\mathbf{A}-\lambda \mathbf{B}) \mathbf{v}\|^{2}}{\|\mathbf{v}\|^{2}}=\underset{\lambda, \mathbf{v} \neq 0}{\operatorname{argmin}} f_{r}(\mathbf{v}, \lambda)
$$

where,

$$
f_{r}(\mathbf{v}, \lambda)=\frac{\left\|\mathbf{R}_{\lambda} \mathbf{v}\right\|^{2}+\mathbf{v}^{*} \mathbf{C} \mathbf{v}}{\|\mathbf{v}\|^{2}}
$$

$\mathbf{R}_{\lambda}=\mathbf{R}_{0}-\lambda \mathbf{R}$ is a parameterized triangular matrix, and $\mathbf{C}$ is Hermitian and positive semidefinite.

Proof. The proof of the above theorem is as follows.

STEP 1. We compute an orthogonal factorization

$$
(\mathbf{B}, \mathbf{A})=\tilde{\mathbf{Q}} \tilde{\mathbf{R}}=\tilde{\mathbf{Q}}\left(\begin{array}{cc}
\mathbf{R}_{11} & \mathbf{R}_{12} \\
\mathbf{O} & \mathbf{R}_{22}
\end{array}\right)
$$

The matrices $\mathbf{R}_{11}, \mathbf{R}_{12}$, and $\mathbf{R}_{22}$ are thus obtained by extracting the $n \times n$ submatrix from the upper left corner, the $n \times n$ sub-matrix from the upper right corner, 
the $(m-n) \times n$ sub-matrix from the lower right corner of the upper triangular matrix $\tilde{\mathbf{R}}$, respectively. We note that $\mathbf{R}_{11}$ is also upper triangular. Using the above decomposition, i.e., equation (3.3), we can write:

$$
\mathbf{A}-\lambda \mathbf{B}=\tilde{\mathbf{Q}}\left(\begin{array}{c}
\mathbf{R}_{12}-\lambda \mathbf{R}_{11} \\
\mathbf{R}_{22}
\end{array}\right)
$$

and, consequently, we get the following intermediate representation:

$$
\|(\mathbf{A}-\lambda \mathbf{B}) \mathbf{v}\|^{2}=\left\|\left(\mathbf{R}_{12}-\lambda \mathbf{R}_{11}\right) \mathbf{v}\right\|^{2}+\left\|\mathbf{R}_{22} \mathbf{v}\right\|^{2} .
$$

STEP 2. To simplify the intermediate representation further, we apply the generalized Schur decomposition (also known as $Q Z$ decomposition) to the matrix pair $\left(\mathbf{R}_{12}, \mathbf{R}_{11}\right)$, to find unitary matrices $\mathbf{Q}, \mathbf{Z}$ such that

$$
\mathbf{R}_{0}=\mathbf{Q R}_{12} \mathbf{Z}, \quad \mathbf{R}=\mathbf{Q R}_{11} \mathbf{Z}
$$

are upper triangular.

STEP 3. Next, we compute the positive semidefinite matrix

$$
\mathbf{C}=\mathbf{E}^{*} \mathbf{E}, \quad \text { where } \quad \mathbf{E}=\mathbf{R}_{22} \mathbf{Z} .
$$

Consequently, we get a reduced form:

$$
\|(\mathbf{A}-\lambda \mathbf{B}) \overline{\mathbf{v}}\|^{2}=\left\|\left(\mathbf{R}_{0}-\lambda \mathbf{R}\right) \overline{\mathbf{v}}\right\|^{2}+\overline{\mathbf{v}}^{*} \mathbf{C} \overline{\mathbf{v}},
$$

where $\overline{\mathbf{v}}$ is related to $\mathbf{v}$ by the following unitary transformation:

$$
\mathbf{v}=\mathbf{Z} \overline{\mathbf{v}} .
$$

Hence we show that it is possible to find a unitary transformation that reduces the problem posed in equation (1.9) with the objective function $f$ to the form stated in equation (3.1) with the objective function $f_{r}$.

REMARK 2. The factor $\tilde{\mathbf{Q}}$ in (3.3) is not required explicitly, which saves storage and computational effort. Thus a Q-less QR-factorization is sufficient for this purpose. The economy version of the LAPACK's QR-factorization routine, or MATLAB's qr function called with the second argument set to ' $0^{\prime}$, returns only the upper triangular matrix.

REMARK 3. We note that the intermediate representation (3.5) is essentially unique if $\mathbf{B}$ has a full rank $n$; but this is not always the case. On the other hand, the above reduction involves only orthogonal transformations, and hence it is stable.

REMARK 4. In the formula (3.5), we note that the first term corresponds to a square eigenvalue problem, while the second term contains the contributions to the noise in a form that is independent of $\lambda$. If the noise is not of interest, or in a noiseless scenario, one may simply discard $\mathbf{R}_{22}$ and proceed with solving the eigenvalue problem:

$$
\mathbf{R}_{12} \mathbf{v}=\lambda \mathbf{R}_{11} \mathbf{v}
$$

using the QZ factorization. In the presence of noise, the solution of the eigenproblem (3.10) does not lead to a solution of the original problem posed in equation (1.9). However, the solution of (3.10) usually gives a good initial guess for the eigenpairs.

REMARK 5. In case $\mathbf{R}_{12}=\mathbf{0}$, the solution to the optimization problem (1.9) is $\lambda=0$, and $\mathbf{v}$ is the left singular vector of $\mathbf{R}_{22}$ corresponding to the smallest singular 
value. In case $\mathbf{R}_{12}$ is close to singular then the value of the expression (2.6) of the condition $\mathbf{C 2}$, Thm 2.1, will turn out to be a significantly large, compared to the case where $\mathbf{R}_{12}$ is well conditioned.

REMARK 6. In equation (3.6), we note that $\mathbf{R}_{11}$ is already triangular while $\mathbf{R}_{12}$ generally is not; thus the preliminary orthogonal factorization and transformation in the QZ-algorithm can be dispensed with. As we shall see later, the above transformation (3.6) significantly reduces complexity.

REMARK 7. Relation (3.9) is used to transform the solution of (3.1) back to the original system (1.9).

REMARK 8. The transformation from equation (1.9) to equation (3.1) requires only the $Q R$ factorization, the $Q Z$ algorithm and the matrix multiplication. All of these steps have a computational complexity of $O\left(m n^{2}\right)$ flops or less. Consequently, the complexity of the reduction of (1.9) to (3.1) is also $O\left(m n^{2}\right)$ flops.

In order to compute the generalized eigenpairs of the matrices $\mathbf{A}$ and $\mathbf{B}$, we first compute the eigenpairs $(\lambda, \mathbf{v})$ of the generalized eigenvalue problem:

$$
\mathbf{R}_{0} \mathbf{v}=\lambda \mathbf{R} \mathbf{v}
$$

or equivalently from equation (3.10). Further we use each of these eigenpairs as a starting point for a local optimization of the objective function in equation (3.2), i.e.,

$$
f_{r}(\mathbf{v}, \lambda)=\frac{\mathbf{v}^{*}\left(\mathbf{R}_{\lambda}^{*} \mathbf{R}_{\lambda}+\mathbf{C}\right) \mathbf{v}}{\mathbf{v}^{*} \mathbf{v}}
$$

Here we use the following notation:

$$
\mathbf{R}_{\lambda}=\mathbf{R}_{0}-\lambda \mathbf{R}
$$

The simple form of the objective functions and the availability of good initial estimates (if the noise term is not too large) allows a direct treatment of the local optimization. At a minimizer, the gradient of (3.1) vanishes, which leads to the following equations:

$$
\left(\mathbf{R}_{\lambda}^{*} \mathbf{R}_{\lambda}+\mathbf{C}\right) \mathbf{v}=\kappa \mathbf{v}
$$

obtained by differentiating the function $f_{r}$ with respect to $\mathbf{v}$, and

$$
(\mathbf{R v})^{*} \mathbf{R}_{\lambda} \mathbf{v}=0
$$

obtained by differentiation the function $f_{r}$ with respect to $\lambda$. Here,

$$
\kappa=f_{r}(\mathbf{v}, \lambda) .
$$

equation (3.14) demonstrates the fact that $\mathbf{v}$ is an eigenvector corresponding to an eigenvalue $\kappa$ of $\mathbf{R}_{\lambda}^{*} \mathbf{R}_{\lambda}+\mathbf{C}$, and since $\kappa=f_{r}(\mathbf{v}, \lambda)$, it must be the smallest eigenvalue.

The left hand side of equation (3.14) is a positive semidefinite Hermitian matrix. One can improve an approximate solution $(\mathbf{v}, \lambda, \kappa)$ by updating $\mathbf{v}$ with an inverse iteration step suggested by equation (3.14), followed by updating $\lambda$ with generalized Rayleigh quotient suggested by equation (3.15), and updating $\kappa$ using equation (3.16). However this approach converges very slowly, and requires $\mathcal{O}\left(n^{3}\right)$ operations per eigenvalue per iteration. 
3.2. Least distance formulation. An efficient algorithm should require only $\mathcal{O}\left(n^{2}\right)$ flops per iteration per eigenpair. To accomplish this objective, within each iteration, we update the generalized eigenvector from $\mathbf{v}$ to $\overline{\mathbf{v}}=\mathbf{v}+\mathbf{d}$. Substituting $\mathbf{v}$ with $\overline{\mathbf{v}}$ in (3.14), and assuming for the moment that $\|\mathbf{d}\|, \kappa,\|\mathbf{C}\|$ are small, we neglect the non-linear terms in $\mathbf{d}, \mathbf{C}$ and $\kappa$, and get the following constraint:

$$
\mathbf{R}_{\lambda}^{*} \mathbf{R}_{\lambda} \overline{\mathbf{v}}-\kappa \mathbf{v}=-\mathbf{C v} .
$$

In order to control the step size $\mathbf{d}$, we obtain the generalized eigenvector $\overline{\mathbf{v}}$ by solving the following least distance problem in the naturally scaled norm:

$$
\begin{aligned}
& \underset{\overline{\mathbf{v}}, \kappa}{\operatorname{argmin}} \quad\left\|\mathbf{R}_{\lambda}(\overline{\mathbf{v}}-\mathbf{v})\right\|^{2} \\
& \text { s.t } \quad \mathbf{R}_{\lambda}^{*} \mathbf{R}_{\lambda} \overline{\mathbf{v}}-\kappa \mathbf{v}=-\mathbf{C v} .
\end{aligned}
$$

We note that (3.18) is a linearly constrained problem with a quadratic objective function; such problems are commonly known as equality constraint quadratic programs. Such programs are solved by solving a linear system, see [9, §5.1.4.2]. The quadratic program (3.18) leads to the following equivalent linear system:

$$
\left(\begin{array}{cc}
\mathbf{R}_{\lambda}^{*} \mathbf{R}_{\lambda} & \mathbf{v} \\
\mathbf{v}^{*} & 0
\end{array}\right)\left(\begin{array}{c}
\overline{\mathbf{v}} \\
-\kappa
\end{array}\right)=\left(\begin{array}{c}
-\mathbf{C v} \\
\mathbf{v}^{*} \mathbf{v}
\end{array}\right)
$$

We note that the LU factorization of the coefficient matrix in the above system of equations (3.19) can be computed in only $\mathcal{O}\left(n^{2}\right)$ operations. However, $\mathbf{R}_{\lambda}$ might be close to singular. Specifically, in the very first iteration if the starting points are computed from equation (3.11). In such a case, one should modify the offending diagonal element. This can be done by replacing the matrix $\mathbf{R}_{\lambda}$ by the matrix

$$
\mathbf{S}=\mathbf{R}_{\lambda}+\rho \mathbf{e}_{\lambda} \mathbf{e}_{\lambda}^{*},
$$

where $\mathbf{e}_{\lambda}=\mathbf{e}^{(k)}$ with $k=\operatorname{argmin}\left|\left(\mathbf{R}_{\lambda}\right)_{k k}\right|$ and $\rho=\max \left|\mathbf{R}_{\lambda}\right|_{j k}$. If the eigenvalues of $\mathbf{R}_{\lambda}$ are reasonably separated (more precisely, if $\mathbf{R}_{\lambda}$ has the numerical rank $n-1$ ) then $\mathbf{S}$ is well-conditioned. We note that the coefficient matrix of (3.19) is a rank 3 perturbation of $\mathbf{S}^{*} \mathbf{S}$. Since $\mathbf{S}$ is triangular, the computational complexity of the algorithm remains $\mathcal{O}\left(n^{2}\right)$ operations per iteration. In case $\|\mathbf{C}\|$ is not too small, the problem of $\mathbf{R}_{\lambda}$ getting close to singular happens only in the very first iteration. Hence another approach to alleviate this problem is by slightly perturbing the initial approximations of $\lambda$ obtained from equation (3.11).

An iterative algorithm to obtain the eigenpairs using the least distance approach is designed as follows: within each iteration update the new eigenvector by solving the system of equations (3.19), and then update the eigenvalue using the generalized Rayleigh quotient:

$$
\bar{\lambda}=\lambda+\frac{(\mathbf{R} \overline{\mathbf{v}})^{*} \mathbf{R}_{\lambda} \overline{\mathbf{v}}}{(\mathbf{R} \overline{\mathbf{v}})^{*} \mathbf{R} \overline{\mathbf{v}}} .
$$

A step by step description of the procedure is given in Algorithm 1

In Section 4 we demonstrate the performance of this algorithm numerically. We observe that the method with least distance formulation, Algorithm 1 takes a lot of iterations to converge unless the initial approximation is very good. Also Algorithm 1 requires $\|\mathbf{C}\|$ and hence $\kappa$ to be small, which might not be the case in presence of significant noise. 


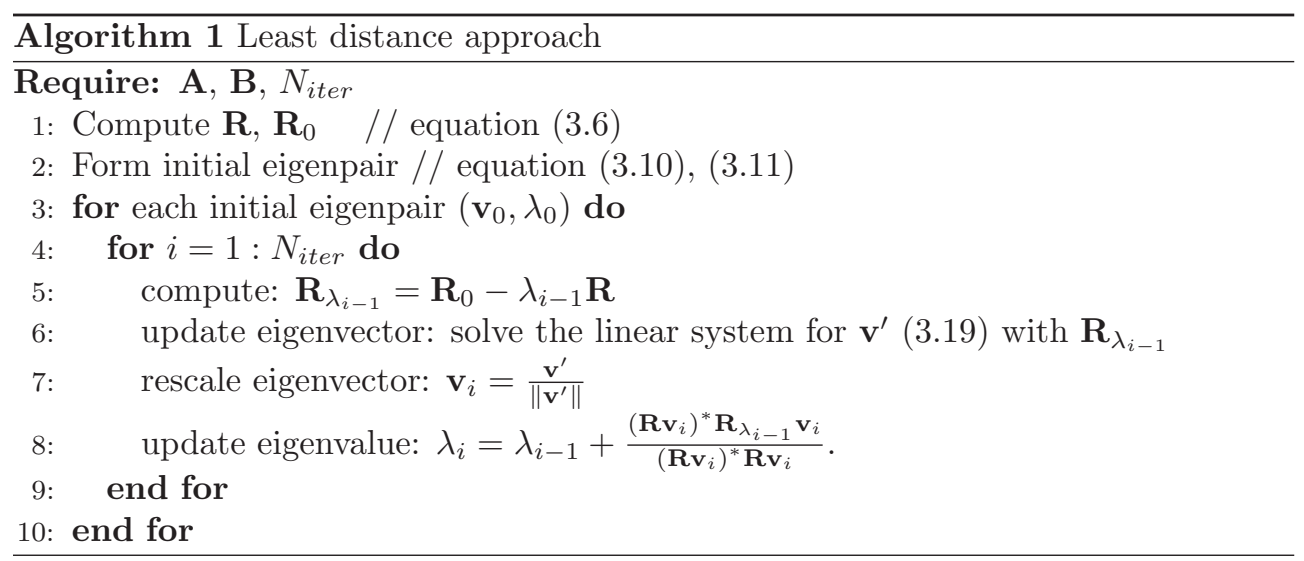

3.3. Joint optimization of eigenvalues and eigenvectors. To achieve a fast convergence, we modify the previous approach with the least distance formulation (3.18), (3.19), in a way that $\lambda$ changes simultaneously with $\mathbf{v}$. We replace $(\mathbf{v}, \lambda)$ in equation (3.14) by $\overline{\mathbf{v}}=\mathbf{v}+\mathbf{d}$ and $\bar{\lambda}=\lambda+\mu$, and obtain the following equation:

$$
\left(\left(\mathbf{R}_{\lambda}-\mu \mathbf{R}\right)^{*}\left(\mathbf{R}_{\lambda}-\mu \mathbf{R}\right)+\mathbf{C}-\kappa \mathbf{I}\right)(\mathbf{v}+\mathbf{d})=0 .
$$

Assuming $\|\mathbf{C}\|$, and hence $\kappa$, are small we neglect all terms nonlinear in $\mathbf{d}, \mu, \kappa$ and $\mathbf{C}$, and find (after reinstating $\overline{\mathbf{v}}=\mathbf{v}+\mathbf{d}$ )

$$
\mathbf{R}_{\lambda}^{*} \mathbf{R}_{\lambda} \overline{\mathbf{v}}-\kappa \mathbf{v}=-\mathbf{C v}+\mu \mathbf{R}_{\lambda}^{*} \mathbf{R} \mathbf{v}+\mu^{*} \mathbf{R}^{*} \mathbf{R}_{\lambda} \mathbf{v} .
$$

The above equation is used only for finding an initial search direction; so it does not matter that the approximate equation (3.23) is not accurate in case $\|\mathbf{C}\|$ is large. The least distance problem (3.18) with the modified constraint (3.23) gives the following system of linear equations:

$$
\left(\begin{array}{cc}
\mathbf{R}_{\lambda}^{*} \mathbf{R}_{\lambda} & \mathbf{v} \\
\mathbf{v}^{*} & 0
\end{array}\right)\left(\begin{array}{c}
\overline{\mathbf{v}} \\
-\kappa
\end{array}\right)=\left(\begin{array}{c}
-\mathbf{C v} \\
\mathbf{v}^{*} \mathbf{v}
\end{array}\right)+\mu\left(\begin{array}{c}
\mathbf{R}_{\lambda}^{*} \mathbf{R} \mathbf{v} \\
0
\end{array}\right)+\mu^{*}\left(\begin{array}{c}
\mathbf{R}^{*} \mathbf{R}_{\lambda} \mathbf{v} \\
0
\end{array}\right) .
$$

Similarly to equation (3.19), the above system of linear equations (3.24) can be solved in $\mathcal{O}\left(n^{2}\right)$ operations. The solution to the above system yields a parametric solution for the eigenvectors in the following form:

$$
\overline{\mathbf{v}}=\mathbf{v}_{0}+\mu \mathbf{v}_{1}+\mu^{*} \mathbf{v}_{2} .
$$

If we denote the inverse of the system matrix in (3.24) by $\mathbf{U}$, i.e.,

$$
\mathbf{U}\left(\begin{array}{cc}
\mathbf{R}_{\lambda}^{*} \mathbf{R}_{\lambda} & \mathbf{v} \\
\mathbf{v}^{*} & 0
\end{array}\right)=\mathbf{I}
$$

then $\mathbf{v}_{0}, \mathbf{v}_{1}, \mathbf{v}_{2}$ are given by truncating the last entry of the vectors

$$
\mathbf{U}\left(\begin{array}{c}
-\mathbf{C v} \\
\mathbf{v}^{*} \mathbf{v}
\end{array}\right), \quad \mathbf{U}\left(\begin{array}{c}
\mathbf{R}_{\lambda}^{*} \mathbf{R} \mathbf{v} \\
0
\end{array}\right), \quad \mathbf{U}\left(\begin{array}{c}
\mathbf{R}^{*} \mathbf{R}_{\lambda} \mathbf{v} \\
0
\end{array}\right)
$$

\footnotetext{
${ }^{1}$ note that $\mathbf{v}_{0}, \mathbf{v}_{1}, \mathbf{v}_{2}$ are not the eigenvectors after initial, first and second iteration
} 
respectively. Because of the nonlinearities one must be prepared to take shorter steps. In order to represent the parametric solution (3.25) with real coefficients, we use:

$$
\overline{\mathbf{v}}=\mathbf{v}+\alpha \mathbf{p}+\beta \mathbf{q}+\gamma \mathbf{r}, \quad \alpha, \beta, \gamma \in \mathbb{R}
$$

where,

$$
\mathbf{p}=\mathbf{v}_{0}-\mathbf{v}, \quad \mathbf{q}=\mathbf{v}_{1}+\mathbf{v}_{2}, \quad \mathbf{r}=i\left(\mathbf{v}_{1}-\mathbf{v}_{2}\right) .
$$

Note that the point $\mathbf{v}$ corresponds to $\alpha=1$, and $\mu=\beta+i \gamma$.

In order to obtain $\alpha, \beta$, and $\gamma$, and to get global convergence even when $\|\mathbf{C}\|$ is large, we substitute $\lambda=\lambda_{0}+\mu$ from equation (3.21) to the objective function in equation (3.2) and get:

$$
f_{1}(\mathbf{v})=\frac{1}{\|\mathbf{v}\|^{2}}\left(\left\|\mathbf{R}_{\lambda_{0}} \mathbf{v}\right\|^{2}-\frac{\left|(\mathbf{R v})^{*}\left(\mathbf{R}_{\lambda_{0}} \mathbf{v}\right)\right|^{2}}{\|\mathbf{R} \mathbf{v}\|^{2}}+\mathbf{v}^{*} \mathbf{C v}\right)
$$

We note that equation (3.30) describes a multi-modal function with minimizers near the eigenvectors of the matrix pencil $\left(\mathbf{R}_{0}, \mathbf{R}\right)$.

Inserting the parametric representation of the eigenvector (3.28) into the function $f_{1}$ in equation (3.30) yields the following rational function of $\alpha, \beta, \gamma$ with explicitly computable coefficients:

$$
f_{p}(\mathbf{g})=\frac{1}{\|\mathbf{V g}\|^{2}}\left(\left\|\mathbf{R}_{\lambda_{0}} \mathbf{V g}\right\|^{2}-\frac{\left|(\mathbf{R V} \mathbf{g})^{*}\left(\mathbf{R}_{\lambda_{0}} \mathbf{V g}\right)\right|^{2}}{\|\mathbf{R V} \mathbf{g}\|^{2}}+\mathbf{g}^{*} \mathbf{V}^{*} \mathbf{C V g}\right)
$$

here we use $\mathbf{g} \equiv[1, \alpha, \beta, \gamma]^{T}$, and $\mathbf{V} \equiv[\mathbf{v}, \mathbf{p}, \mathbf{q}, \mathbf{r}]$. This function can be optimized in $\mathcal{O}(1)$ operations to get the optimal values of $\alpha, \beta, \gamma$. It is easy to see that each iteration $\mathbf{v} \rightarrow \overline{\mathbf{v}}$ gives a strict improvement to $f_{1}(\mathbf{v})$ unless $\mathbf{v}$ is already optimal, and can be computed in $\mathcal{O}\left(n^{2}\right)$ operations. A step by step description of the algorithm based on the joint optimization of $\lambda$ and $\mathbf{v}$ is given in Algorithm 2 .

REMARK 9. The parameter $\alpha$ always converge to 1 . However, the value of $\alpha$ should not be fixed to 1 over the iterations. The parameter $\alpha$ denotes the step size in the direction of the last estimate of the eigenvector ( $\mathbf{v})$ towards the least distance solution $\left(\mathbf{v}_{0}\right)$. Fixing $\alpha=1$ will force the algorithm to take the full step towards that direction, and therefore successive estimates of the eigenvalue (specifically initial few iterations) will not follow a smooth path. Consequently, if the initial guess of the eigenvalue is not too close to the actual value then fixing alpha $=1$ increases the likelihood of missing the actual eigenvalue. But if we optimize on $\alpha$, then successive estimates of the eigenvalue follow a smooth path and finally converges to the actual eigenvalue.

4. Numerical experiments. In this section we demonstrate our approach numerically. At first we present the simulation setup that we used for the numerical experiments. Next we compare our approach with the MPA. In the next subsection we report the performance of the proposed algorithms in different scenarios. In the final subsection, we demonstrate how the proposed method is beneficial for computing the distance to uncontrollability of a linear control system.

A MATLAB based implementation of the proposed algorithms can be downloaded from :

http://www.mat.univie.ac.at/ neum/software/oeig/

This software is used for the numerical experiments given in this section. 


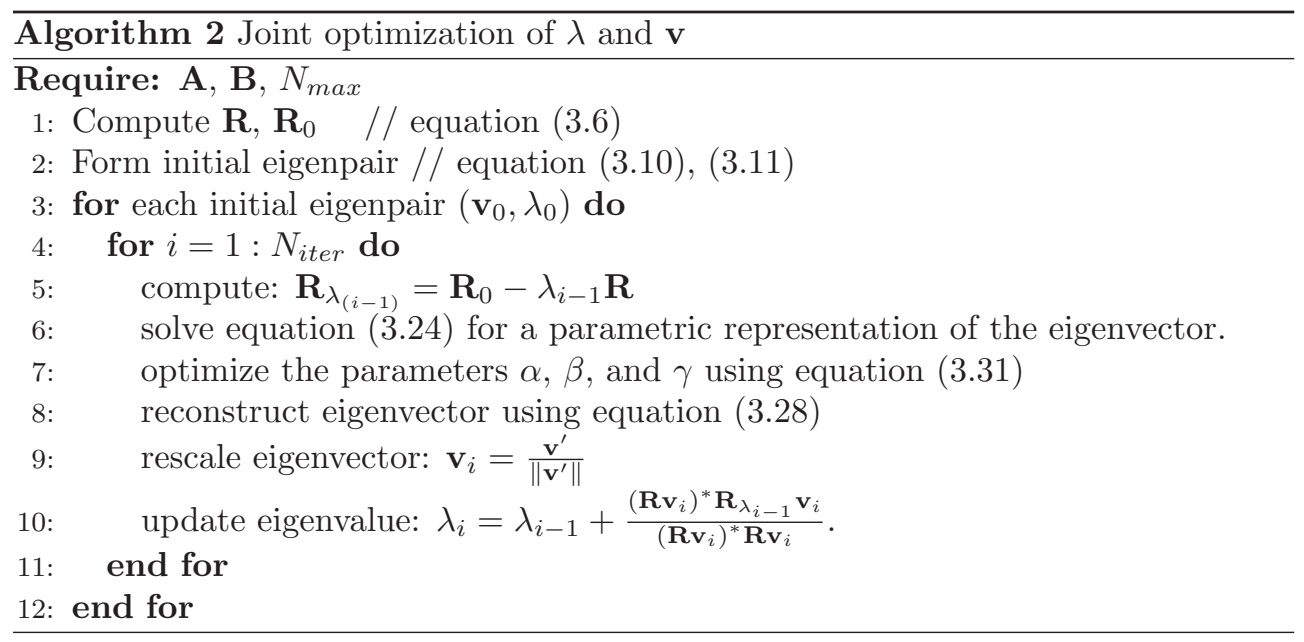

4.1. Simulation setup. We simulate overdetermined matrices for testing the performance of the proposed algorithms. Our objective is to simulate overdetermined noisy matrix pairs $(\mathbf{A}, \mathbf{B})$ of size $m \times n$ that might not admit any non-trivial solution, but the unperturbed noiseless counterpart of this matrix pair, say $\left(\mathbf{A}_{0}, \mathbf{B}_{0}\right)$, admits only $r$ non-trivial eigenvalues $\lambda_{1}, \ldots, \lambda_{r}$, where $r \leqslant n$.

We first generate a pair of square matrices $\left(\mathbf{A}_{s}, \mathbf{B}_{s}\right)$ of size $n \times n$ such that $\lambda_{1}, \ldots, \lambda_{r}$ are the only non-trivial generalized eigenvalues of this matrix pair. Such matrix pairs are easy to construct. We set $\mathbf{A}_{s}$ to an upper triangular matrix with the first $r$ diagonal entries set to $\lambda_{1}, \ldots, \lambda_{r}$ and the remaining diagonal entries set to 1 , all other entries above diagonal are random. We set $\mathbf{B}_{s}$ to another upper triangular matrix with the first $r$ diagonal entries set to 1 and the remaining diagonal entries set to 0 , all other entries above diagonal are random. We note that the super diagonal entries of $\mathbf{A}_{s}$ and $\mathbf{B}_{s}$ can be used to adjust the conditioning of the matrices and hence their susceptibility to noise. Further, we multiply both the matrices $\mathbf{A}_{s}$ and $\mathbf{B}_{s}$ from left and right with a pair of unitary matrices, which also results in a matrix pair, say $\left(\mathbf{A}_{q}, \mathbf{B}_{q}\right)$, with $\lambda_{1}, \ldots, \lambda_{r}$ as the only generalized eigenvalues. Next, we multiply both $\mathbf{A}_{q}$ and $\mathbf{B}_{q}$ from the left with an $m \times n$ matrix of full rank, i.e., $n$. The products form a matrix pair that has $\lambda_{1}, \ldots, \lambda_{r}$ as the only generalized eigenvalues. These products are the desired noiseless matrices $\left(\mathbf{A}_{0}, \mathbf{B}_{0}\right)$, which admit only $r$ specified non-trivial eigenvalues $\lambda_{1}, \ldots, \lambda_{r}$. Finally, we perturb each entry of the matrix pair $\left(\mathbf{A}_{0}, \mathbf{B}_{0}\right)$ with complex additive noise to get the desired matrix pair $(\mathbf{A}, \mathbf{B})$. With such simulated matrix pairs, the backward stability of any algorithm for overdetermined eigenvalue problem can be studied easily.

4.2. Comparison with the MPA. In this section, we compare the proposed optimization problem posed in equation (1.9) and (1.10), with the MPA optimization problem posed in equation (1.6) and (1.7). We observe that for certain kind of matrix pairs that frequently arises in practice, the factor $1 / \sqrt{1+|\lambda|^{2}}$ in the objective function (1.7) of the MPA has the effect that the eigenvalues of relatively large magnitude go unnoticed. In order to illustrate this fact, we simulate a matrix pair $\left(\mathbf{A}_{0}, \mathbf{B}_{\mathbf{0}}\right)$ of dimension $50 \times 5$, which admits only one non-trivial solution $\lambda=9$.0. Further, we perturbed each entry of the matrix pair $\left(\mathbf{A}_{0}, \mathbf{B}_{\mathbf{0}}\right)$ with additive Gaussian noise (with $\sigma=.01)$ to obtain the matrix pair $(\mathbf{A}, \mathbf{B})$. We evaluate the objective function of the 

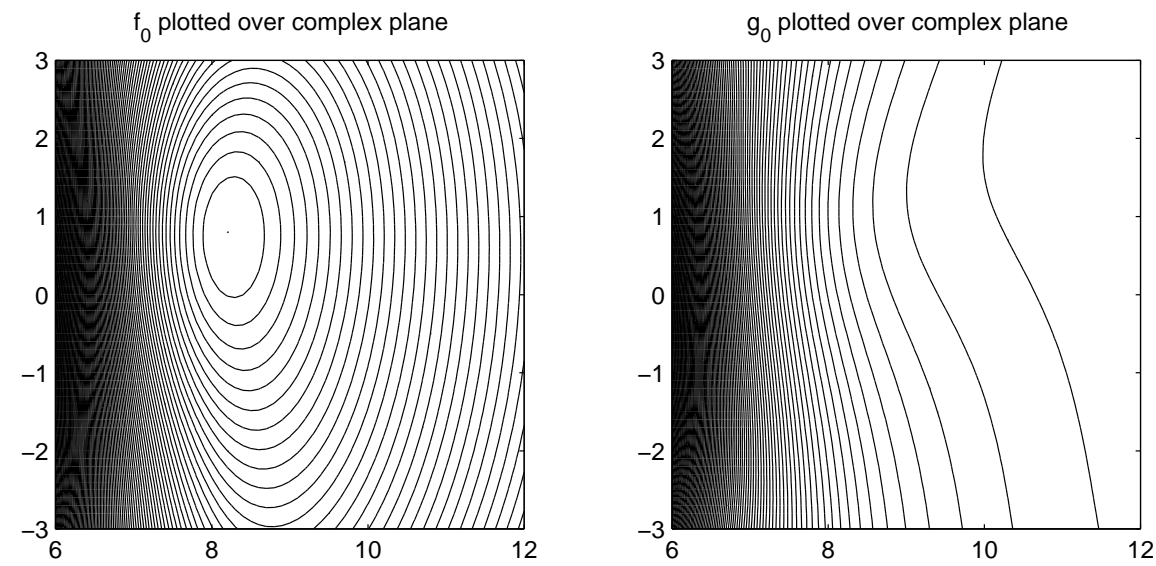

FIG. 4.1. Level curves of the function $f_{0}$ and $g_{0}$ in the complex plane

problem we solve as posed in equation (1.10), and the objective function of the MPA as posed in equation (1.7) over a fine grid in the complex plane. The level curves of both the functions are plotted in Figure 4.1. We note that for the objective function $f_{0}$ in equation (1.10) corresponding to our approach, a minima close to 9.0 is visible, and one can expect a solution. On the other hand, the function $g_{0}$ in equation (1.7), corresponding to the MPA, does not show any sign of a local minimum around 9.0. This observation is explained by the fact that in the above example, $\sigma_{\min }(\mathbf{A}-\lambda \mathbf{B})$ is increasing slower than $\sqrt{1+|\lambda|^{2}}$ around the local minimum at $\lambda=9.0$.

We note that for a matrix pair $\left(\mathbf{A}_{0}, \mathbf{B}_{\mathbf{0}}\right)$ with exactly $n$ generalized eigenvalues, the surface $\sigma_{\min }\left(\mathbf{A}_{0}-\lambda \mathbf{B}_{0}\right)$ increases to infinity outside a certain finite disc in the complex plane. On the other hand, for a matrix pair $\left(\mathbf{A}_{0}, \mathbf{B}_{\mathbf{0}}\right)$ with a smaller number of generalized eigenvalues than the dimension of the domain, i.e., $r<n$, the surface $\sigma_{\min }\left(\mathbf{A}_{0}-\lambda \mathbf{B}_{0}\right)$ decreases to zero outside a certain finite disc in the complex plane. Thus, especially for matrix pairs where $r<n$, the MPA is not sufficient to locate the generalized eigenvalues. Moreover, even if $g_{0}$, the objective function of the MPA, locates the minima properly, the surface around the minima is very flat because of the factor $1 / \sqrt{1+|\lambda|^{2}}$, which in turn slows down the optimization procedure. In 3], all the numerical experiments were done using matrix pair $(\mathbf{A}, \mathbf{B})$ of size $m \times n$, which were obtained by perturbing matrix pair $\left(\mathbf{A}_{0}, \mathbf{B}_{\mathbf{0}}\right)$, admitting $n$ generalized eigenpairs. However, practical problems where $\left(\mathbf{A}_{0}, \mathbf{B}_{\mathbf{0}}\right)$ admits fewer eigenpairs than $n$ is not uncommon. For example, such a matrix pair is obtained in a harmonic inversion problem, when the assumed model order for estimation is greater than the number of harmonic components present in the signal.

We note that, apart from the problems of fading eigenvalues and slow convergence, the MPA based algorithm requires $\mathcal{O}\left(n^{3}\right)$ operation per iteration per eigenpair. On the other hand, the proposed method requires $\mathcal{O}\left(n^{3}\right)$ operation per iteration per eigenpair.

4.3. Results. In order to illustrate the performance of the proposed algorithms numerically, we simulate a matrix pair $\left(\mathbf{A}_{0}, \mathbf{B}_{0}\right)$ of size $15 \times 5$ that has only the following three non-trivial eigenvalues $2+4 i, 3+2 i$, and $4+2.2 i$. Further, we generate a matrix pair $(\mathbf{A}, \mathbf{B})$ by perturbing each entry of the matrix pair $\left(\mathbf{A}_{0}, \mathbf{B}_{0}\right)$ with complex additive Gaussian white noise of standard deviation $\sigma=.01$. In Figure 4.2 we show the noise-free (exact) eigenvalues; the starting eigenvalues; the final estimate with 
the joint optimization method (Algorithm 2); and the level curve of the function $f_{0}$ over the complex plane. We observe that the estimated eigenvalues converged close to the exact noise-free eigenvalues. The gap that remains between the exact noise-free eigenvalues and the estimated ones is due to the noise in the matrix pairs $(\mathbf{A}, \mathbf{B})$. One can expect that with more data points, i.e., with more rows in the matrix pairs $(\mathbf{A}, \mathbf{B})$ this gap is diminished, and thereby a better estimation of the eigenvalues is achieved. In order to demonstrate this fact, we simulated several matrix pair $(\mathbf{A}, \mathbf{B})$ each with 5 columns and varying number of rows, using the same eigenvalues for the noiseless counterpart, and same level of noise $(\sigma=.01)$ to perturb the entries. In Figure 4.3(a), we report the root mean square error (RMSE) of the estimated eigenvalues with respect to the noise-free eigenvalues computed over 1000 random perturbation of the noise-free matrix pair $\left(\mathbf{A}_{0}, \mathbf{B}_{0}\right)$. For matrix pairs of size $5 \times 5$ the eigenvalues were estimated using the $Q \mathrm{Z}$ decomposition. In Figure 4.3(a) we observe that the estimation of the eigenvalues gets more accurate with increasing number of rows. The next experimental setup is similar to the previous one, except that we fix the size of the matrix pair to $30 \times 5$, and vary the intensity of the additive noise. In Figure 4.3(b) we report the root mean square error (RMSE) of the estimated eigenvalues with respect to the noise-free eigenvalues computed over 1000 random perturbation of the noise-free matrix pair $\left(\mathbf{A}_{0}, \mathbf{B}_{0}\right)$ with different intensities of the additive white Gaussian noise.

In the following paragraphs we discuss the rate of convergence. Rather than giving a theoretical proof, we use numerical experiments to demonstrate the rate of convergence. For the $i$ th iteration, we define the distance to the exact solution $e_{i}$ (or the error), the quotient of the error $q_{i}$, and the root of the error $r_{i}$ as follows:

$$
\begin{gathered}
e_{i}=\left|\lambda_{i}-\lambda_{\infty}\right| \\
q_{i}=\frac{e_{i+1}}{e_{i}}=\frac{\left|\lambda_{i+1}-\lambda_{\infty}\right|}{\left|\lambda_{i}-\lambda_{\infty}\right|} . \\
r_{i}=\sqrt[i]{e_{i}}=\sqrt[i]{\left|\lambda_{i}-\lambda_{\infty}\right|} .
\end{gathered}
$$

Here, $\lambda_{i}$ is the estimate of the eigenvalue in the $i$ th iteration, and $\lambda_{\infty}$ is the final estimate, i.e., result of the last iteration. The limiting values of $q_{i}$ and $r_{i}$ should demonstrate the rate of convergence.

We consider the pair $(\mathbf{A}, \mathbf{B})$ each of size $30 \times 5$ with the same noiseless eigenvalues used in the last illustration, i.e., $2+4 i, 3+2 i$, and $4+2.2 i$. Each entry of the noiseless matrices are perturbed with additive white Gaussian noise of standard deviation $\sigma=.01$. In Figure 4.4 we show the quotient of the error $r_{i}$ and the root of the error $r_{i}$ of the eigenvalues obtained with the method based on joint optimization of eigenvalues and eigenvectors, i.e., Algorithm 2, We notice that the method converges very fast within first 10 iterations.

The algorithm based on a least distance approach, i.e., Algorithm 1 converges much slower compared to the algorithm based on joint optimization of eigenvalues and eigenvectors, i.e., Algorithm 2, Starting from the same initial eigenvalues, as shown in Figure 4.2 Algorithm 1 requires several thousands of iterations to converge, which might be prohibitive for practical application.

We found that the MPA based approach also requires several thousands of iterations to convergence. In Figure 4.5 we show the error as defined in equation (4.1) at 


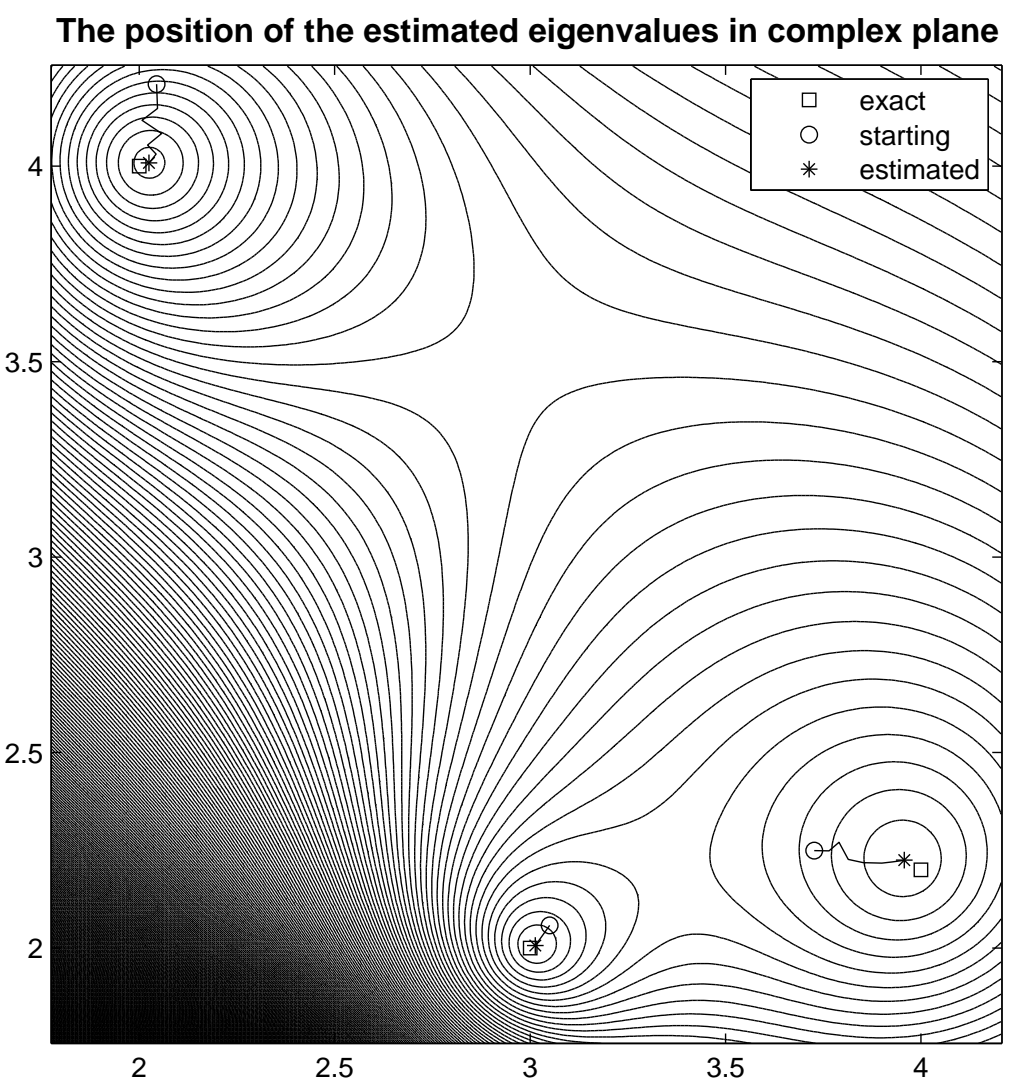

FIG. 4.2. The complex plane showing the noiseless (exact) eigenvalue, the starting eigenvalue, the estimated eigenvalue, and the path traced over the iterations.

each iteration, as obtained using Algorithm 2 and the MPA based method. The same pair of matrices of size $30 \times 5$ as described above were used, with the same starting points as shown in Figure 4.2. We note that the MPA based method converges relatively fast when it gets very close to the solution. However, it is not easy to find such an accurate starting point with noisy matrix pairs. On several examples, we observed that the MPA approach converges relatively faster only in case the eigenvalues are close to zero, and the number of eigenvalues is equal to the dimension of the domain, i.e., $n$.

Depending on the method in which the data is collected, the rows of the matrix pair (A, B) might not be homogeneous. Considering the fact that we use Euclidean norm in equation (1.9), the matrix pair might require a pre-processing step of row equilibration to obtain a meaningful result.

4.4. Distance to Uncontrollability. In Section 1, we described how the problem of estimating distance to uncontrollability is related to the overdetermined eigenvalue problem. In this paragraph, we show the results obtained using Algorithm 2 on two linear control problems. The first problem is an example of an uncontrollable system; with this example we verify that the proposed algorithm when used for computing the distance to uncontrollability can detect uncontrollable systems. We consider the problem given as Example 2 in [10, §4]. Using Algorithm 2 we find that 


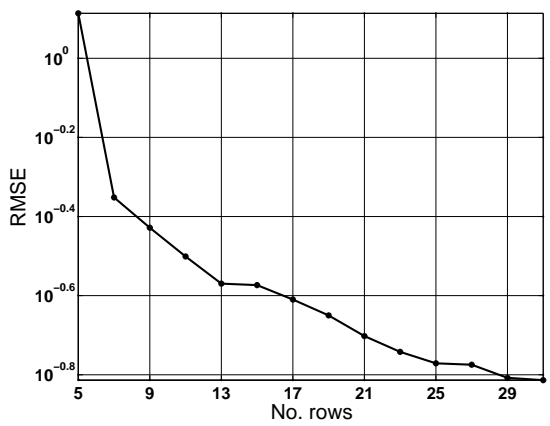

(a) Dependence of accuracy on the number of rows

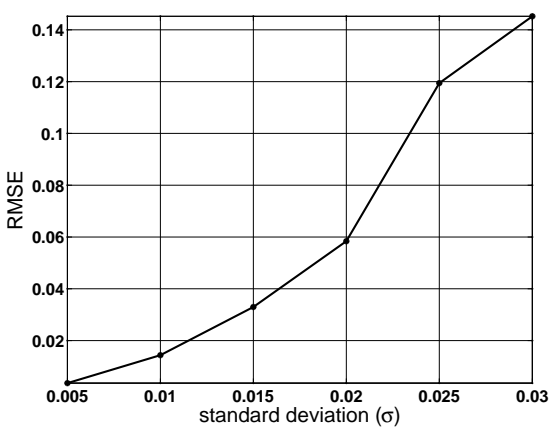

(b) Dependence of accuracy on the additive noise level

FIG. 4.3. Estimation using joint optimization of eigenvalue and eigenvector, Algorithm 2

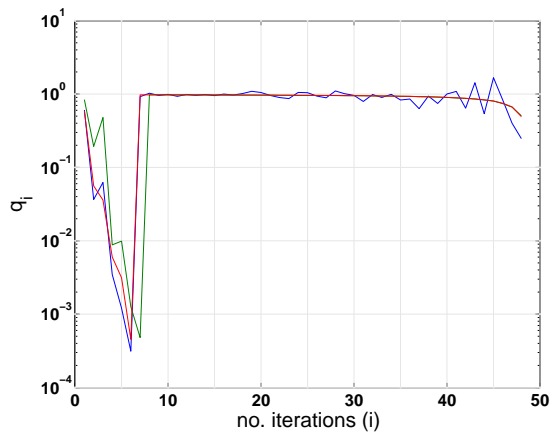

(a) q-rate

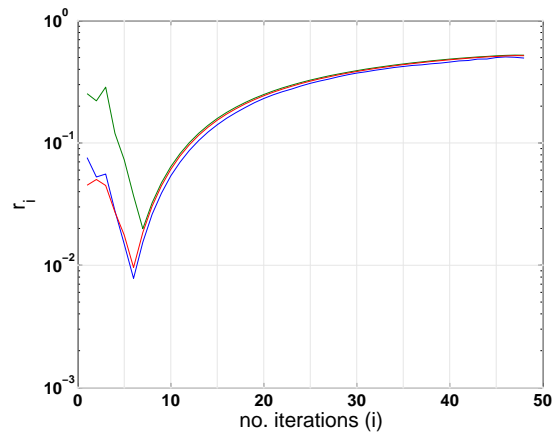

(b) r-rate

FIG. 4.4. Rate of convergence of Algorithm 2

for this system the distance to uncontrollability is 0 , i.e., this system is an example of an uncontrollable system. The second problem is a controllable system; hence for this example we compute the distance to uncontrollability. For this purpose, we consider the problem given as Example 1 in [12, §6]. Using Algorithm 2 we find that the distance to uncontrollability is $3.923843021870004 E-2$. This result matches with the one published in [12] with 14 correct digits. In fact, the proposed algorithm computes much more spectral information which is discarded when only the distance to uncontrollability is required.

The fastest algorithm available so far for computing the distance to uncontrollability is proposed by Gu in [11. The method proposed in [11] has a computational complexity of $\mathcal{O}\left(n^{4}\right)$. On the other hand, the proposed method computes in practice all the eigenvalues with $\mathcal{O}\left(n^{3}\right)$ computations, though not with a theoretical guarantee. Consequently, the proposed method can be employed in practice to compute the distance to uncontrollability with $\mathcal{O}\left(n^{3}\right)$ computations.

5. Relation to pseudospectra. Pseudospectra (see 24]) help to visualize and understand the spectral properties of matrices, especially for non-normal matrices. The $\epsilon$-pseudospectrum of a square matrix $\mathbf{A}$ is defined as

$$
\Lambda_{\epsilon}(\mathbf{A})=\left\{\lambda \in \mathbb{C}: \sigma_{\min }(\mathbf{A}-\lambda \mathbf{I}) \leqslant \epsilon\right\},
$$




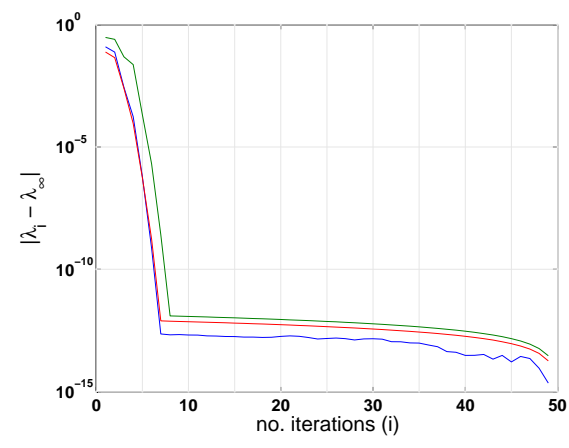

(a) Algorithm 2

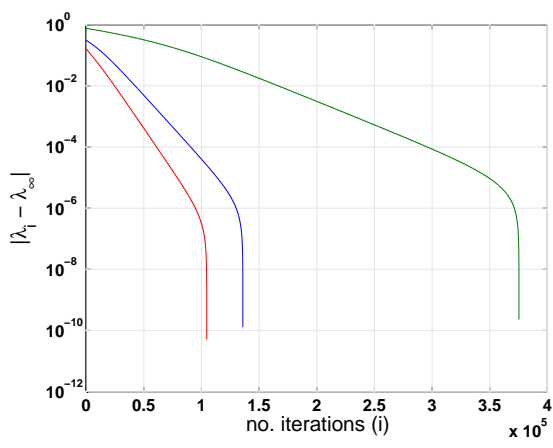

(b) algorithm based on the MPA

FIG. 4.5. Estimation using Algorithm 2 and algorithm based on the minimal perturbation approach (MPA) [3]. Three curves in each plot corresponds to three generalized eigenvalues of the matrix pairs.

where $\sigma_{\min }$ returns the smallest singular value of its matrix argument. While there are other equivalent definitions of pseudospectra, we choose this particular definition (5.1) because of its close relation with our definition of overdetermined eigenvalues. According to [24. Eq. (2)], the pseudospectrum is a set that contains the eigenvalues as well as eigenvalues of a perturbed system, where the perturbation is bounded by $\epsilon$.

Pseudospectra for matrix pencils are introduced in [25]. The $\epsilon$-pseudospectrum of a pair of square matrices $\mathbf{A}, \mathbf{B}$ is defined as follows:

$$
\Lambda_{\epsilon}(\mathbf{A}, \mathbf{B})=\left\{\lambda \in \mathbb{C}: \sigma_{\min }(\mathbf{A}-\lambda \mathbf{B}) \leqslant \epsilon\right\},
$$

Pseudospectra for non-square (rectangular) matrices are described in [26]. As discussed in [26, the $\epsilon$-pseudospectrum of a pair of matrices $\mathbf{A}, \mathbf{B}$ with perturbation bounded by $\epsilon$ in the matrix $\mathbf{A}$ and no perturbation in the matrix $\mathbf{B}$ is again given by equation (5.2).

Our definition of overdetermined eigenvalues matches with the definitions of the pseudospectrum given in equations (5.2). From the pseudospectrum point of view, our definition of overdetermined eigenvalues gives point in the complex plane from where a connected component of the pseudospectrum begins to grow as $\epsilon$ is increased from zero.

In [26, §6.1] the $\epsilon$-pseudospectrum when both the matrices are perturbed, such that the perturbation in $\mathbf{A}$ is bounded by $\alpha \epsilon$ and the perturbation in $\mathbf{B}$ is bounded by $\beta \epsilon$, is reported as follows:

$$
\Lambda_{\epsilon}(\mathbf{A})=\left\{\lambda \in \mathbb{C}: \frac{\sigma_{\min }(\mathbf{A}-\lambda \mathbf{B})}{\alpha+\beta|\lambda|} \leqslant \epsilon\right\} .
$$

On the other hand, the analysis presented in $[3$ suggests the following $\epsilon$-pseudospectrum

$$
\Lambda_{\epsilon}(\mathbf{A}, \mathbf{B})=\left\{\lambda \in \mathbb{C}: \frac{\sigma_{\min }(\mathbf{A}-\lambda \mathbf{B})}{\sqrt{\alpha+\beta|\lambda|^{2}}} \leqslant \epsilon\right\} .
$$

Although our definition of overdetermined eigenvalues (1.10) corresponds to pseudospectra of rectangular matrices where only one matrix is perturbed, we obtained very satisfactory overdetermined eigenvalues even when both the matrices are perturbed. See $\S$ 团 for details of the experiments performed. 
6. Conclusions. In this paper we proposed methods for the solution of the generalized eigenvalue problems $(\mathbf{A}-\lambda \mathbf{B}) \mathbf{v} \approx 0$ for overdetermined matrices $\mathbf{A}$ and B. Our approach is based on searching for $\lambda$ which minimizes the smallest singular value over the matrix pencil $\{\mathbf{A}-\lambda \mathbf{B}\}$. We derived conditions under which the overdetermined eigenpairs are stable. For estimating the overdetermined eigenpairs, we developed two algorithms. The first one was designed as an algorithm with $\mathcal{O}\left(n^{2}\right)$ operations per iteration per eigenvalue, hence a least distance formulation approach is used. This approach was found to be very slow if the initial approximations are away from the solution. In order to alleviate this problem, a second, improved method was developed, where the eigenvectors and eigenvalues are optimized simultaneously. The second method locates the solution very fast, and requires $\mathcal{O}\left(n^{2}\right)$ operations per iteration. In our experiments we found that the final method requires less than 20 iterations to converge.

The proposed method improves in several ways upon the algorithm based on the minimal perturbation approach (MPA). The proposed method requires $\mathcal{O}\left(n^{2}\right)$ operations per iterations per eigenvalue, while the MPA based method requires $\mathcal{O}\left(n^{3}\right)$ for the same task. The proposed method converges much faster than the MPAbased method. Moreover, the objective function used by the proposed method seems more appropriate than the one used in MPA, since the later tends to lose meaningful overdetermined eigenvalues.

\section{REFERENCES}

[1] F. Alharbi, Meshfree eigenstate calculation of arbitrary quantum well structures, Physics Letters A (2010).

[2] D. Boley, Estimating the sensitivity of the algebraic structure of pencils with simple eigenvalue estimates, SIAM J. Matrix Anal. Appl 11 (1990), no. 4, 632-643.

[3] G. Boutry, M. Elad, G.H. Golub, and P. Milanfar, The generalized eigenvalue problem for nonsquare pencils using a minimal perturbation approach, SIAM Journal on Matrix Analysis and Applications 27 (2006), no. 2, 582-601.

[4] A.M. Dell, RL Weil, and GL Thompson, Roots of Matrix Pencils, Communications of the Association for Computing Machinery 14 (1971), 113-117.

[5] A. Edelman, E. Elmroth, and B. Kågström, A geometric approach to perturbation theory of matrices and matrix pencils. Part I: Versal deformations, SIAM Journal on Matrix Analysis and Applications 18 (1997), no. 3, 653-692.

[6] R. Eising, Between controllable and uncontrollable, Systems \& control letters 4 (1984), no. 5, 263-264.

[7] E. Elmroth, P. Johansson, and B. Kågström, Bounds for the distance between nearby Jordan and Kronecker structures in a closure hierarchy, Journal of Mathematical Sciences 114 (2003), no. 6, 1765-1779.

[8] L. Elsner and C. He, An algorithm for computing the distance to uncontrollability, Systems \& control letters 17 (1991), no. 6, 453-464.

[9] P.E. Gill, W. Murray, and M.H. Wright, Practical optimization, Academic press, 1981.

[10] M. Gu, New methods for estimating the distance to uncontrollability, SIAM Journal on Matrix Analysis and Applications 21 (2000), no. 3, 989-1003.

[11] M. Gu, E. Mengi, ML Overton, J. Xia, and J. Zhu, Fast methods for estimating the distance to uncontrollability, SIAM journal on matrix analysis and applications 28 (2007), no. 2, $477-502$.

[12] C. He, Estimating the distance to uncontrollability: A fast method and a slow one, Systems \& control letters 26 (1995), no. 4, 275-281.

[13] N.J. Higham, M. Konstantinov, V. Mehrmann, and P. Petkov, The sensitivity of computational control problems, Control Systems Magazine, IEEE 24 (2004), no. 1, 28-43.

[14] Y. Hua and T.K. Sarkar, Matrix pencil method for estimating parameters of exponentially damped/undamped sinusoids in noise, Acoustics, Speech and Signal Processing, IEEE Transactions on 38 (1990), no. 5, 814-824.

[15] _ On SVD for estimating generalized eigenvalues of singular matrix pencil in noise, 
Signal Processing, IEEE Transactions on 39 (2002), no. 4, 892-900.

[16] CKE Lau, RS Adve, and TK Sarkar, Combined CDMA and matrix pencil direction of arrival estimation, Vehicular Technology Conference, 2002. Proceedings. VTC 2002-Fall. 2002 IEEE 56th, vol. 1, IEEE, 2002, pp. 496-499.

[17] P. Lecumberri, M. Gómez, and A. Carlosena, Generalized Eigenvalues of Nonsquare Pencils with Structure, SIAM Journal on Matrix Analysis and Applications 30 (2008), 41.

[18] C.B. Moler and G.W. Stewart, An algorithm for generalized matrix eigenvalue problems, SIAM Journal on Numerical Analysis 10 (1973), no. 2, 241-256.

[19] S.P. Neugebauer, A Deterministic Blind Equalization Method for Multi-Modulus Signals, Sensor Array and Multichannel Processing, 2006. Fourth IEEE Workshop on, IEEE, 2008, pp. $551-555$.

[20] H. Ouibrahim, Prony, Pisarenko, and the matrix pencil: a unified presentation, Acoustics, Speech and Signal Processing, IEEE Transactions on 37 (2002), no. 1, 133-134.

[21] R. Richard and K. Thomas, ESPRIT-estimation of signal parameters via rotational invariance techniques, IEEE Trans on Acoustics and Signal processing 37 (1989), no. 7, 984-995.

[22] T.K. Sarkar and O. Pereira, Using the matrix pencil method to estimate the parameters of a sum of complex exponentials, Antennas and Propagation Magazine, IEEE 37 (2002), no. 1, $48-55$.

[23] GW Stewart, Perturbation theory for rectangular matrix pencils, Linear Algebra and its Applications 208 (1994), 297-301.

[24] L.N. Trefethen, Pseudospectra of linear operators, Siam Review (1997), 383-406.

[25] J.L.M. Van Dorsselaer, Pseudospectra for matrix pencils and stability of equilibria, BIT Numerical Mathematics 37 (1997), no. 4, 833-845.

[26] T.G. Wright and L.N. Trefethen, Pseudospectra of rectangular matrices, IMA Journal of Numerical Analysis 22 (2002), no. 4, 501. 